\title{
Surgical management of acute esophageal necrosis
}

\author{
Grigoriy E. Gurvits
}

Received: 1 April 2013/Accepted: 21 May 2013/Published online: 31 January 2014

(C) Springer Japan 2014

I read with great interest the article by Drs. M.H. Wu and H.Y. Wu [1] on acute esophageal necrosis (AEN). The authors described two patients with AEN and emphasized the role of esophagectomy in the treatment of this rare syndrome. While I welcome reports of new cases of "black esophagus" that stir scientific discussion about the condition, I disagree with the proposed, rather aggressive, surgical approach to manage AEN. In my original paper, I introduced a staging system that describes the progression of AEN based on the endoscopic and pathologic findings [2]. It should be noted that black esophagus is a striking medical condition that may present with acute gastrointestinal hemorrhage in patients with hemodynamic compromise, debilitated health, sepsis, multiorgan failure, acute gastric outlet obstruction and diabetic ketoacidosis. The mainstay of treatment of AEN is supportive medical therapy with acid suppression, nil-per-os restriction, red blood cell transfusion, and importantly, correction of the underlying medical condition that has led to the development of the AEN. The overall mortality rate in patients with black esophagus is high at $31 \%$, but the mortality specific to the esophageal disease is only $6 \%$. To date, surgical resection has been reserved only for cases of esophageal perforation, an event typically caused by external compression by a ruptured thoracic aorta, gastric volvulus or severe thromboembolic disease [3]. It may also be considered in situations with rapid clinical decompensation in the absence of any other explanation. I absolutely disagree with the authors that esophagectomy is indicated for a wide group of stage 1 AEN cases. I further disagree that surgical intervention is necessary for stages 2 and 3 of AEN to prevent the development of esophageal strictures. A subgroup of patients with diabetes mellitus has a low chance of developing stricture or stenotic complications, and all patients generally do well with endoscopic balloon dilatation [2].

In conclusion, the surgical management of AEN should be performed only in select cases of perforation/mediastinitis. The passage of a nasogastric tube, and especially a Sengstaken-Blakemore tube, in AEN patients should be prohibited, as the friability of the esophagus will lead to tragic perforation and death, as was seen in the second patient. In their description of their two cases of AEN, the authors failed to explain the need for rapid surgical treatment of patients with black esophagus, without first attempting conventional medical management.

Conflict of interest The author has no commercial associations that might be a conflict of interest in relation to the submitted manuscript.

\section{References}

1. Wu MH, Wu HY. Incremental changes in acute esophageal necrosis: report of two cases. Surg Today. 2013;44(2):363-5.

2. Gurvits GE, Shapsis A, Lau N, Gualtieri N, Robilotti JG. Acute esophageal necrosis: a rare syndrome. J Gastroenterol. 2007;42:29-38.

3. Gurvits GE. Black esophagus: acute esophageal necrosis syndrome. World J Gastroenterol. 2010;16:3219-25.
G. E. Gurvits $(\square)$

Department of Gastroenterology, New York University Medical Center, 530 First Ave, SKI-9N, New York, NY 10016, USA

e-mail: g_gurvits@hotmail.com 